\title{
The classification of 231-avoiding permutations by descents and maximum drop
}

\author{
Matthew Hyatt AND Jeffrey Remmel
}

\begin{abstract}
We study the number of 231-avoiding permutations of length $n$ with $j$-descents and maximum drop of less than or equal to $k$, which we denote by $a_{n, j}^{(k)}$. We show that $a_{n, j}^{(k)}$ also counts the number of Dyck paths of length $2 n$ with $n-j$ peaks and height $\leq k+1$, and the number of ordered trees with $n$ edges, $j+1$ internal nodes, and height $\leq k+1$. We show that the generating functions for the $a_{n, j}^{(k)} \mathrm{s}$ with $k$ fixed satisfy a simple recursion. We also use the combinatorics of ordered trees to prove new explicit formulas for $a_{n, j}^{(k)}$ and prove a simple recursion for the $a_{n, j}^{(k)} \mathrm{s}$.

AMS 2000 SUBJECT ClASSIFICATIONS: Primary 05A15; secondary 05 E05. Keywords AND PHRASEs: Permutation statistics, 231-avoiding permutations, descents, drops, trees, Dyck paths.
\end{abstract}

\section{Introduction}

In [1], Chung, Claesson, Dukes, and Graham studied generating functions for permutations according to the number of descents and the maximum drop. Here if $\sigma=\sigma_{1} \ldots \sigma_{n}$ is a permutation in the symmetric group $S_{n}$, then we say that $\sigma$ has drop at $i$ if $\sigma_{i}<i$ and $\sigma$ has a descent at $i$ if $\sigma_{i}>\sigma_{i+1}$. MacMahon proved that the number of permutations with $k$ descents is equal to the number of permutations with $k$ drops. Let $[n]=\{1,2, \ldots, n\}$ and for any $1 \leq i<j \leq n$, let $[i, j]=\{s \in[n]: i \leq s \leq j\}$. We let

$$
\operatorname{DES}(\sigma)=\left\{i \in[n]: \sigma_{i}>\sigma_{i+1}\right\}, \quad \operatorname{des}(\sigma)=|\operatorname{DES}(\sigma)|,
$$

and

$$
\operatorname{maxdrop}(\sigma)=\max \left\{i-\sigma_{i}: i \in[n]\right\} .
$$

We let $\mathcal{B}_{n}^{(k)}$ denote the set of permutations $\sigma \in S_{n}$ such that $\operatorname{maxdrop}(\sigma) \leq$ $k$.

There is another interpretation of $\mathcal{B}_{n}^{(k)}$ in terms of the classic bubble sort, which we denote by bubble. (See [8] for more on the bubble sort operator.)

arXiv: 1208.1052 
Let $\operatorname{bsc}(\sigma)=\min \left\{i: \operatorname{bubble}^{i}(\sigma)=\right.$ id $\}$, i.e. $\operatorname{bsc}(\sigma)$ is the minimum number of times that bubble must be applied to $\sigma$ in order to reach the identity permutation. An inductive argument shows that $\operatorname{bsc}(\sigma)=\operatorname{maxdrop}(\sigma)$, thus $\mathcal{B}_{n}^{(k)}$ is the set of permutations in $S_{n}$ which can be sorted by applying bubble $k$ times. Additionally, the permutations in $\mathcal{B}_{n}^{(k)}$ are in bijective correspondence with certain juggling sequences (see [1]).

Let

$$
B_{n}^{(k)}(x)=\sum_{\sigma \in \mathcal{B}_{n}^{(k)}} x^{\operatorname{des}(\sigma)}=\sum_{j=0}^{n-1} b_{n, j}^{(k)} x^{j},
$$

where $b_{n, j}^{(k)}$ denotes the number permutations in $S_{n}$ (not necessarily avoiding the pattern 231) with $j$-descents, and maximum drop less than or equal to $k$. Note that for $k \geq n-1, \mathcal{B}_{n}^{(k)}=S_{n}$ and $B_{n}^{(k)}(x)$ becomes the Eulerian polynomial

$$
B_{n}(x)=\sum_{\sigma \in S_{n}} x^{\operatorname{des}(\sigma)} .
$$

For convenience we let $B_{0}(x)=1$. In [1], the authors prove the following generating function formula

$$
\begin{aligned}
B^{(k)}(x, t) & =\sum_{n \geq 0} B_{n}^{(k)}(x) t^{n} \\
& =\frac{1+\sum_{r=1}^{k}\left(B_{r}(x)-\sum_{i=1}^{r}\left(\begin{array}{c}
k+1 \\
i
\end{array}\right)(x-1)^{i-1} B_{r-i}(x)\right) t^{r}}{1-\sum_{i=1}^{k+1}\left(\begin{array}{c}
k+1 \\
i
\end{array}\right) t^{i}(x-1)^{i-1}} .
\end{aligned}
$$

We now turn our attention to pattern avoidance. Given a sequence $\sigma=\sigma_{1} \ldots \sigma_{n}$ of distinct integers, let $\operatorname{red}(\sigma)$ be the permutation found by replacing the $i$-th smallest integer that appears in $\sigma$ by $i$. For example, if $\sigma=2754$, then $\operatorname{red}(\sigma)=1432$. Given a permutation $\tau=\tau_{1} \ldots \tau_{j}$ in the symmetric group $S_{j}$, we say that the pattern $\tau$ occurs in $\sigma=\sigma_{1} \ldots \sigma_{n} \in S_{n}$ provided there exists $1 \leq i_{1}<\cdots<i_{j} \leq n \operatorname{such}$ that $\operatorname{red}\left(\sigma_{i_{1}} \ldots \sigma_{i_{j}}\right)=\tau$. We say that a permutation $\sigma$ avoids the pattern $\tau$ if $\tau$ does not occur in $\sigma$. Let $S_{n}(\tau)$ denote the set of permutations in $S_{n}$ which avoid $\tau$. In the theory of permutation patterns (see [6] for a comprehensive introduction to the area), $\tau$ is called a classical pattern. Throughout the rest of this paper we focus on permutations avoiding the pattern 231 . Thus we let

$$
\mathcal{A}_{n}^{(k)}=S_{n}(231) \cap \mathcal{B}_{n}^{(k)} .
$$


Thus $\mathcal{A}_{n}^{(k)}$ is the set of $\sigma \in S_{n}$ such that $\operatorname{maxdrop}(\sigma) \leq k$ and $\sigma$ avoids 231. The set $\mathcal{A}_{n}^{(k)}$ can also be interpreted in terms of sorting algorithms. The 231-avoiding permutations are precisely the permutations which can be sorted by one application of the stack sort, which we denote by stack (see $[7])$. So

$$
\mathcal{A}_{n}^{(k)}=\left\{\sigma \in S_{n}: \operatorname{bubble}^{k}(\sigma)=\mathrm{id}, \text { and } \operatorname{stack}(\sigma)=\mathrm{id}\right\},
$$

i.e. the permutations in $S_{n}$ which can be sorted by one stack sort, but require at most $k$ bubble sorts to be sorted.

The main goal of this paper is to study the generating functions

$$
A^{(k)}(x, t)=1+\sum_{n \geq 1} A_{n}^{(k)}(x) t^{n}
$$

where

$$
A_{n}^{(k)}(x)=\sum_{\sigma \in \mathcal{A}_{n}^{(k)}} x^{\operatorname{des}(\sigma)}=\sum_{j=0}^{n-1} a_{n, j}^{(k)} x^{j} .
$$

Note that the only permutation $\sigma \in S_{n}$ such that $\operatorname{maxdrop}(\sigma)=0$ is the identity permutation $\sigma=123 \ldots n$ which is 231 -avoiding. Thus $A_{n}^{(0)}(x)=1$ for all $n \geq 1$ so that

$$
A^{(0)}(x, t)=\frac{1}{1-t} .
$$

Our key theorem is to show that generating functions $A^{(k)}(x, t)$ for $k \geq 1$ satisfy the following simple recursion.

Theorem 1. For all $k \geq 1$,

$$
A^{(k)}(x, t)=\frac{1}{1-t+t x-t x A^{(k-1)}(x, t)}
$$

where

$$
A^{(0)}(x, t)=\frac{1}{1-t} .
$$

Theorem 1 allowed us to explicitly compute the values of $a_{n, j}^{(k)}$ for small values of $j, k$, and $n$, which lead us to conjecture and prove a number of simple formulas for $a_{n, j}^{(k)}$ in certain special cases. For example, one can show that

$$
a_{n, 1}^{(k)}=\left(\begin{array}{l}
n \\
2
\end{array}\right)
$$


for all $n \geq 2$ and $k \geq 1$, and

$$
a_{n, 2}^{(k)}=\frac{(n-1)^{2}\left((n-1)^{2}-1\right)}{12}
$$

for $n \geq 3$ and $k \geq 2$. More generally, we shall show that for all $n>j \geq 1$ and all $k \geq j$,

$$
a_{n, j}^{(k)}=N(n, n-j)=\frac{1}{n}\left(\begin{array}{c}
n \\
j
\end{array}\right)\left(\begin{array}{c}
n \\
j+1
\end{array}\right) .
$$

Here the $N(n, j)$ s are the Narayana numbers which count the number of Dyck paths of length $2 n$ with $j$ peaks, and also count the number of ordered trees $n$ edges and $j$ leaves. This suggested that the numbers $a_{n, j}^{(k)}$ should also have natural combinatorial interpretations in terms of Dyck paths and ordered trees. In fact, we construct bijections to show that $a_{n, j}^{(k)}$ is the number of ordered trees with height less than or equal to $k+1, n$ edges, and $j+1$ internal nodes and is the number of Dyck paths of length $2 n$ with $n-j$ peaks and height less than or equal to $k+1$.

Kemp [5] gave a general formula for the number of ordered trees with $n$ edges, $j$ leaves, and height less than or equal to $k$, which we denote by $N(n, j, k)$ (which is also the number of Dyck paths of length $2 n$ with $j$ peaks and height less than or equal to $k$ ). Thus we have a general formula for $a_{n, j}^{(k)}$. However, in many cases, Kemp's formula is unnecessarily complicated so that we use the combinatorics of ordered trees to derive a new recurrence for the numbers $N(n, j, k)$ and $a_{n, j}^{(k)}$.

Theorem 2. For all $k \geq 2$ and for all $n \geq j \geq 1$ we have

$$
a_{n, n-j}^{(k-1)}=N(n, j, k)=\sum_{i=0}^{n-j} N(n-j, i, k-1)\left(\begin{array}{c}
2 n-j-i \\
2 n-2 j
\end{array}\right),
$$

where $N(n, j, 1)=1$ if $n=j$, and is zero otherwise.

We note that the recurrence in Theorem 2 is different from the recurrence implied by Theorem 1. By iterating the recurrence in Theorem 2, we obtain an explicit formula for $N(n, j, k)$ which looks strikingly different than the formula of Kemp. Specifically, the formula below is a positive sum of products of binomials coefficients, whereas Kemp's formula contains negative terms.

Corollary 1. For $n \geq j \geq 0$ and $k \geq 3$ we have 


$$
N(n, j, k)=a_{n, n-j}^{(k-1)}=\sum_{n-j \geq i_{k-2} \geq \cdots \geq i_{1} \geq 0}\left(\prod_{m=0}^{k-2}\left(\begin{array}{c}
i_{m+2}+i_{m} \\
2 i_{m+1}
\end{array}\right)\right),
$$

where $i_{0}:=0, \quad i_{k-1}:=n-j, \quad i_{k}:=n$.

Remark 1. Our attempts to simplify the expression on the right hand side of Corollary 1 have been unsuccessful. An interesting problem would be to simplify this expression in a meaningful way.

The outline of this paper is as follows. In section 2, we shall prove Theorem 1 as well as provide a combinatorial proof of the fact that $a_{n, r}^{(1)}=\left(\begin{array}{c}n \\ 2 r\end{array}\right)$ for all $r \geq 1$ and $n \geq 2 r$. In section 3, we shall prove our alternative combinatorial interpretations of $a_{n, j}^{(k)}$ in terms of ordered trees and Dyck paths. In section 4 , we shall use the combinatorics of ordered trees to prove formulas for $a_{n, j}^{(k)}$, and provide the proofs of Theorem 2 and Corollary 1. Finally, in section 5 , we shall briefly discuss some combinatorial identities that arise by comparing Kemp's formula and our formulas.

\section{Proof of Theorem 1}

In this section, we shall prove Theorem 1 . We accomplish this by first proving the following claim for $n \geq 2$,

$$
A_{n}^{(k)}(x)=A_{n-1}^{(k)}(x)+x \sum_{i=1}^{n-1} A_{i-1}^{(k)}(x) A_{n-i}^{(k-1)}(x) .
$$

The proof proceeds by decomposing the 231-avoiding permutations $\sigma=$ $\sigma_{1} \ldots \sigma_{n}$ by the position of $n$ in $\sigma$. Classically each $\sigma \in S_{n}(231)$ has the structure pictured in Figure 1. That is, in the graph of $\sigma$, the elements to the left of $n, L_{i}(\sigma)$, have the structure of a 231-avoiding permutation, the elements to the right of $n, R_{i}(\sigma)$, have the structure of a 231-avoiding permutation, and all the elements in $L_{i}(\sigma)$ lie below all the elements in $R_{i}(\sigma)$. Note that the number of 231-avoiding permutations in $S_{n}$ is the Catalan number $C_{n}=\frac{1}{n+1}\left(\begin{array}{c}2 n \\ n\end{array}\right)$ and the generating function for the $C_{n}$ 's is given by

$$
C(t)=\sum_{n \geq 0} C_{n} t^{n}=\frac{1-\sqrt{1-4 t}}{2 t}=\frac{2}{1+\sqrt{1-4 t}} .
$$

Suppose that $k \geq 1$ and $n \geq 2$. Let $\mathcal{A}_{n}^{(k)}(i)$ denote the set of $\sigma \in \mathcal{A}_{n}^{(k)}$ such that $\sigma_{i}=n$. Clearly if $\sigma \in \mathcal{A}_{n}^{(k)}(i)$, then $L_{i}(\sigma)$ must be a permutation in 


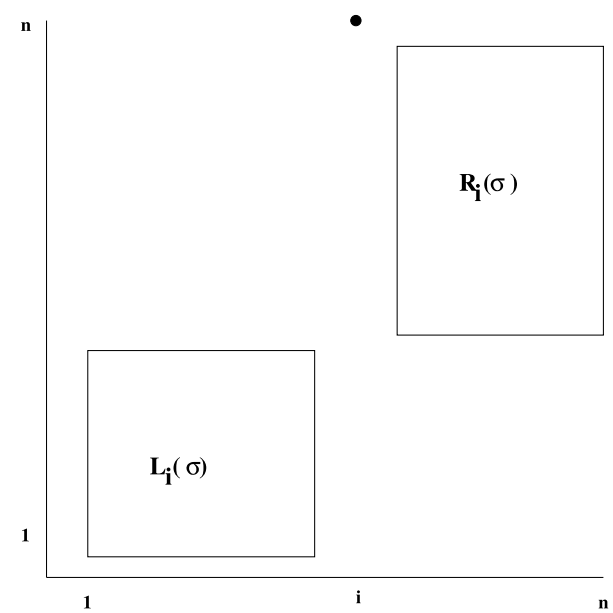

Figure 1: The structure of 231-avoiding permutations.

$S_{i-1}(231)$ such that maxdrop $\left(L_{i}(\sigma)\right) \leq k$. Similarly, $\tau=\operatorname{red}\left(R_{i}(\sigma)\right)$ must be a permutation in $S_{n-i}(231)$ such that $\operatorname{maxdrop}(\tau) \leq k-1$. That is, we can consider $R_{i}(\sigma)$ as a map from $\{(i+1, \ldots, i+(n-i)\}$ into $\{(i-1+1, \ldots,(i-$ $1)+(n-i)\}$. Thus for $j=1, \ldots, n-i$, a drop $j-\tau_{j}$ in $\tau$ corresponds to a drop $i+j-\left(i-1+\tau_{j}\right)=i+j-\sigma_{i+j}-1$ in $\sigma$. Thus the drop at position $j$ in $\tau$ is one less than the drop at position $i+j$ in $\sigma$. Now if $i \leq n-1$, then $\sigma_{i}=n$ will start a descent in $\sigma$. Thus the possible choices for $L_{i}(\sigma)$ will contribute a factor of $A_{i-1}^{(k)}(x)$ to $\sum_{\sigma \in \mathcal{A}_{n}^{(k)}(i)} x^{\operatorname{des}(\sigma)}$ and the possible choices for $R_{i}(\sigma)$ will contribute a factor of $A_{n-i}^{(k-1)}(x)$ to $\sum_{\sigma \in \mathcal{A}_{n}^{(k)}(i)} x^{\operatorname{des}(\sigma)}$. Thus the contribution of the permutations in $\mathcal{A}_{n}^{(k)}(i)$ to $A_{n}^{(k)}(x)$ is $x A_{i-1}^{(k)}(x) A_{n-i}^{(k-1)}(x)$. Finally, it is easy to see that the contribution of the permutations in $\mathcal{A}_{n}^{(k)}(n)$ to $A_{n}^{(k)}(x)$ is just $A_{n-1}^{(k)}(x)$. The claim in (4) follows from these facts.

Note that $A_{1}^{(k)}(x)=1$ so that if we define $A_{0}^{(k)}(x)=1$, then (4) also holds for $n=1$. Multiplying both sides of (4) by $t^{n}$ and summing for $n \geq 1$, we see that for $k \geq 1$,

$$
\begin{aligned}
A^{(k)}(x, t)-1 & =t A^{(k)}(x, t)+t \sum_{n \geq 1} t^{n-1} x \sum_{i=1}^{n-1} A_{i-1}^{(k)}(x) A_{n-i}^{(k-1)}(x) \\
& =t A^{(k)}(x, t)+t x A^{(k)}(x, t)\left(A^{(k-1)}(x, t)-1\right) .
\end{aligned}
$$

Solving this equation for $A^{(k)}(x, t)$, we see that 


$$
A^{(k)}(x, t)=\frac{1}{1-t+x t-x t A^{(k-1)}(x, t)},
$$

which proves Theorem 1.

One can use Mathematica to calculate the first few of the generating functions $A_{231}^{(k)}(x, t)$.

$$
\begin{aligned}
& A^{(0)}(x, t)=\frac{1}{1-t}, \\
& A^{(1)}(x, t)=\frac{1-t}{1-2 t+(1-x) t^{2}}, \\
& A^{(2)}(x, t)=\frac{1-2 t+(1-x) t^{2}}{1-3 t+(3-2 x) t^{2}-(1-x)^{2} t^{3}}, \\
& A^{(3)}(x, t)=\frac{1-3 t+(3-2 x) t^{2}-(1-x)^{2} t^{3}}{1-4 t+3(2-x) t^{2}-2\left(2-3 x+x^{2}\right) t^{3}+(1-x)^{3} t^{4}}, \\
& A^{(4)}(x, t)=\frac{1-4 t+3(2-x) t^{2}-2\left(2-3 x+x^{2}\right) t^{3}+(1-x)^{3} t^{4}}{1-5 t+(10-4 x) t^{2}-\left(10-12 x+3 x^{2}\right) t^{3}-(1-x)^{2}(2 x-5) t^{4}-(1-x)^{4} t^{5}} .
\end{aligned}
$$

By setting $x=1$, we can obtain information on the asymptotic growth rate of the cardinality of $\mathcal{A}_{n}^{(k)}$. For $k=0,1$, it is easy to extract the exact value of $\left|\mathcal{A}_{n}^{(k)}\right|$ for all $n$, namely $\left|\mathcal{A}_{n}^{(0)}\right|=1$ and $\left|\mathcal{A}_{n}^{(1)}\right|=2^{n-1}$. For $k=$ $2,3,4$, we give the exponential growth rate of $\left|\mathcal{A}_{n}^{(k)}\right|$. Following Flajolet and Sedgewick [2], let

$$
\left|\mathcal{A}_{n}^{(k)}\right| \bowtie K^{n} \quad \text { if and only if } \quad \limsup _{n \rightarrow \infty}\left|\mathcal{A}_{n}^{(k)}\right|^{1 / n}=K .
$$

By examining the modulus of the poles ([2, Theorem IV.7]), we find that

$$
\left|\mathcal{A}_{n}^{(2)}\right| \bowtie\left(\frac{3+\sqrt{5}}{2}\right)^{n}, \quad\left|\mathcal{A}_{n}^{(3)}\right| \bowtie 3^{n}, \quad\left|\mathcal{A}_{n}^{(4)}\right| \bowtie(3.247)^{n} .
$$

Note that the denominator of $A^{(4)}(1, t)$ is a cubic, and we have given a numeric approximation of the reciprocal of the modulus of the root closest to the origin.

It turns out that all the coefficients of the generating function $A^{(1)}(x, t)$ are binomial coefficients. Since $\left|\mathcal{A}_{n}^{(1)}\right|=2^{n-1}$, this suggested a bijective explanation for the coefficients of $A^{(1)}(x, t)$.

Indeed, let $\mathcal{P}([n])$ denote the set of all subsets of $[n]$, and $\mathcal{P}_{e}([n])$ denote the set of all elements of $\mathcal{P}([n])$ that have even cardinality. We define a 
$\operatorname{map} \phi: \mathcal{P}_{e}([n]) \rightarrow \mathcal{A}_{n}^{(1)}$ as follows. First, let $\phi(\emptyset)=12 \ldots n$. Now if $S=$ $\left\{s_{1}, s_{2}, \ldots, s_{2 r-1}, s_{2 r}\right\} \in \mathcal{P}_{e}([n])$ where $1 \leq s_{1}<s_{2}<\cdots<s_{2 r-1}<s_{2 r} \leq n$, then we consider the intervals $I_{j}=\left[s_{2 j-1}, s_{2 j}\right]$ for $j=1, \ldots, r$. We define

$$
\phi(S)=\tau^{S}=\tau_{1}^{S} \ldots \tau_{n}^{S}
$$

to be the permutation in $S_{n}$ such that $\tau_{i}^{S}=i$ if $i$ is not in one of the intervals $I_{1}, \ldots, I_{r}$, and

$$
\tau_{s_{2 j-1}}^{S} \ldots \tau_{s_{2 j}}^{S}=s_{2 j} s_{2 j-1}\left(s_{2 j-1}+1\right) \ldots\left(s_{2 j}-1\right) .
$$

For example, if $n=12$, and $S=\{1,3,6,8,10,12\}$, then $I_{1}=[1,3], I_{2}=$ $[6,8]$, and $I_{3}=[10,12]$. Thus

$$
\tau^{S}=312458679121011
$$

Theorem 3. For all $r \geq 1$ and $n \geq 2 r$, the map $\phi: \mathcal{P}_{e}([n]) \rightarrow \mathcal{A}_{n}^{(1)}$ is a bijection satisfying $|S|=2 \operatorname{des}(\phi(S))$. Consequently

$$
\left.A_{n}^{(1)}(x)\right|_{x^{r}}=a_{n, r}^{(1)}=\left(\begin{array}{c}
n \\
2 r
\end{array}\right) .
$$

Proof. On each of the intervals $I_{j}, \tau^{S}$ has maximum drop 1 so that $\operatorname{maxdrop}\left(\tau^{S}\right) \leq 1$ for all $S \in \mathcal{P}_{e}([n])$. Moreover it easy to see that $\tau^{S}$ is 231 avoiding and that we can recover $S$ from $\tau^{S}$. Thus $\phi$ is a one-to-one map from $\mathcal{P}_{e}([n])$ into $\mathcal{A}_{n}^{(1)}$. However, since we know that $\left|\mathcal{P}_{e}([n])\right|=2^{n-1}=\left|\mathcal{A}_{n}^{(1)}\right|$, the map $\phi$ must also be a surjection. Thus $\phi$ is a bijection from $\mathcal{P}_{e}([n])$ onto $\mathcal{A}_{n}^{(1)}$.

Since each interval $I_{j}$ introduces exactly one descent in $\tau^{S}$, it follows that for all $S \in \mathcal{P}_{e}([n])$, we have $\operatorname{des}(\phi(S))=\frac{|S|}{2}$. Thus the number of $\sigma \in \mathcal{A}_{n}^{(1)}$ such that $\operatorname{des}(\sigma)=r$ equals the number of subsets $S$ of $[n]$ of size $2 r$. That is, $\left.A_{n}^{(1)}(x)\right|_{x^{r}}=\left(\begin{array}{c}n \\ 2 r\end{array}\right)$.

\section{Ordered trees of bounded height}

We begin this section by showing there is a bijective correspondence between permutations in $S_{n}(231)$ with a given maximum drop and a given number of descents, to a certain class of trees. An ordered tree is a rooted tree where the children of each vertex are ordered, so for example we can refer to the leftmost child of a vertex. We use the convention of placing the root at the top of the tree. Micheli and Rossin show there is a bijection between 231-avoiding permutations and ordered trees [9]. Given an ordered 

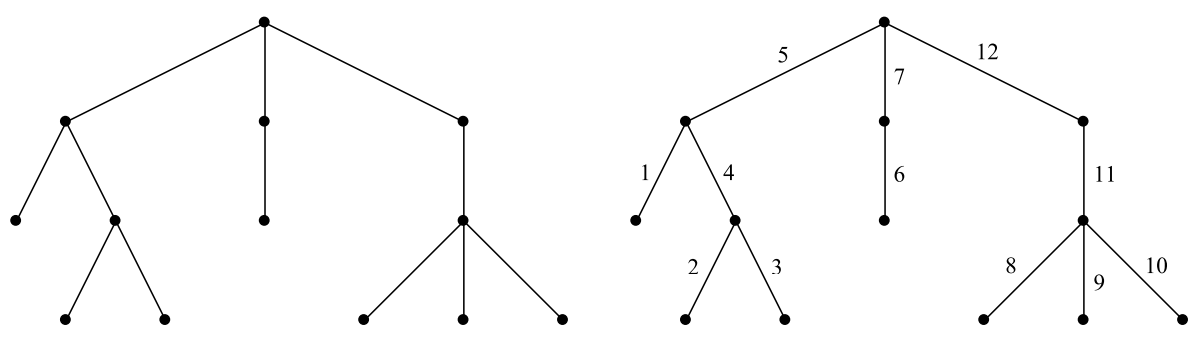

Figure 2: An ordered tree $T$ (left), $T$ with edges labeled by a postorder traversal (right).

tree $T$, let $\phi(T)$ be the word obtained by first labeling the edges of $T$ by a postorder traversal, then reading the labels by a preorder traversal. For example, consider the ordered tree $T$ in Figure 2. When we read the labels by a preorder traversal, we obtain the permutation $\phi(T)$ (which we write in two-line notation)

$$
\phi(T)=\left[\begin{array}{lllllllccccc}
1 & 2 & 3 & 4 & 5 & 6 & 7 & 8 & 9 & 10 & 11 & 12 \\
5 & 1 & 4 & 2 & 3 & 7 & 6 & 12 & 11 & 8 & 9 & 10
\end{array}\right] .
$$

We show that this same bijection also carries additional information about the descents and maximum drop of 231-avoiding permutations. The level of a vertex is the length of the unique path from that vertex to the root. The height of an ordered tree is the maximum of the levels of all vertices in the tree. An internal node is a vertex which has at least one child. Let $\mathcal{T}_{n, j}^{(k)}$ denote the set of all ordered trees having $n$ edges, height less than or equal to $k$, and $j$ internal nodes. Let $\mathcal{A}_{n, j}^{(k)}$ denote the set of permutations in $\sigma \in S_{n}(231)$ with $\operatorname{des}(\sigma)=j$ and $\operatorname{maxdrop}(\sigma) \leq k$, thus $\left|\mathcal{A}_{n, j}^{(k)}\right|=\left.A_{n}^{(k)}(x)\right|_{x^{j}}=a_{n, j}^{(k)}$.

Theorem 4. The map $\phi: \mathcal{T}_{n, j}^{(k)} \rightarrow \mathcal{A}_{n, j-1}^{(k-1)}$ is a bijection for all $n, k, j \geq 1$, thus

$$
a_{n, j}^{(k)}=\left|\mathcal{T}_{n, j+1}^{(k+1)}\right| .
$$

In other words $a_{n, j}^{(k)}$ is equal to the number of ordered trees with $n$ edges, $j+1$ internal nodes, and height less than or equal to $k+1$.

Proof. Given $T \in \mathcal{T}_{n, j}^{(k)}$, let $\phi(T)=\sigma$. Since Micheli and Rossin showed that $\sigma \in S_{n}(231)$ and $\phi$ is a bijection (see [9]), our Theorem is proved if we can show that $\operatorname{des}(\sigma)=j-1$ and $\operatorname{maxdrop}(\sigma) \leq k-1$. 


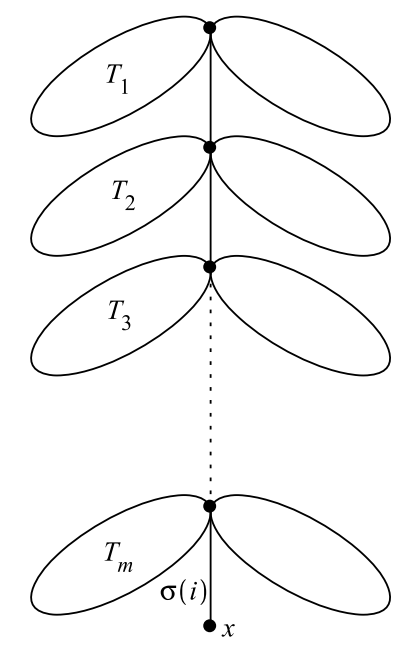

Figure 3: An ordered tree with edge labeled $\sigma_{i}$ directly above a leaf $x$ at level $m$.

First we show that $\operatorname{des}(\sigma)=j-1$. Given any edge of $T$, let $\sigma_{i}$ be its label from the postorder traversal, and let $x$ be the vertex at the bottom of this edge.

If $x$ is an internal node, then $\sigma_{i+1}$ is the label on the leftmost edge immediately below $x$. Since the labeling is done with a postorder traversal, we have $\sigma_{i}>\sigma_{i+1}$.

If $x$ is not an internal node (i.e. a leaf), then either it is the case that $x$ is the rightmost leaf of $T$ in which case the label on the edge is $\sigma_{n}$ or there is a vertex $y$ with subtrees $Y_{1}$ and $Y_{2}$ below $y$ such that $\sigma_{i}$ is a label on an edge of $Y_{1}, \sigma_{i+1}$ is a label on an edge of $Y_{2}$, and $Y_{1}$ is to the left of $Y_{2}$ so that $\sigma_{i}<\sigma_{i+1}$.

Since every vertex other than the root is at the bottom of a unique edge, $\sigma$ has $j-1$ descents.

Next we show that maxdrop $(\sigma) \leq k-1$. Suppose $\sigma_{i}<i$, and let $x$ be the vertex at the bottom of the edge labeled $\sigma_{i}$.

If $x$ is an internal node, then $\sigma_{i}>\sigma_{i+1}$ as noted above, thus there is a larger drop size at position $i+1$ in $\sigma$. Since we want to find the maximum drop size, we need only consider the case that $x$ is a leaf. Thus assume that $x$ is a leaf and let $m$ be the level of $x$. On the path from $x$ to the root, there are $m$ (possibly empty) subtrees along the left side of the path, as in Figure 3. Let $\left|T_{r}\right|$ denote the number of edges in a tree $T_{r}$. Then we have 


$$
\left|T_{1}\right|+\left|T_{2}\right|+\cdots+\left|T_{m}\right|=\sigma_{i}-1,
$$

since the edges in the subtrees $T_{1}, T_{2}, \ldots, T_{m}$ are precisely the edges which precede the edge labeled $\sigma_{i}$ in the postorder traversal. The edges in the subtrees $T_{1}, T_{2}, \ldots, T_{m}$ along with the edges in the path from $x$ to the root are precisely the edges which precede the edge labeled $\sigma_{i}$ in the preorder traversal, therefore

$$
i=\left|T_{1}\right|+\left|T_{2}\right|+\cdots+\left|T_{m}\right|+m=\sigma_{i}+m-1 .
$$

Thus $\sigma$ has a drop of size $i-\sigma_{i}=m-1$ at position $i$. Since $m \leq k$, we have $\operatorname{maxdrop}(\sigma) \leq k-1$.

The set of trees in $\mathcal{T}_{n, j}^{(k)}$ are also in bijection with certain Dyck paths. A Dyck path of length $2 n$ is a path in the plane that starts at the point $(0,0)$ and ends at the point $(2 n, 0)$. The path may consist only of up-steps $(1,1)$ and down-steps $(1,-1)$, and the path always stays on or above the $x$-axis. Let $\mathcal{D}_{2 n}$ denote the set of Dyck paths of length $2 n$. Next we describe a couple statistics for Dyck paths. The height of a Dyck path is the highest $y$-coordinate attained in the path. A peak is a point in a Dyck path which is immediately preceded by an up-step, and immediately followed by a downstep. Let $\mathcal{D}_{2 n, j}^{(k)}$ denote the set of Dyck paths of length $2 n$ with $j$ peaks and height less than or equal to $k$. The standard bijection from ordered trees to Dyck paths preserves height, and converts each leaf to a peak. A tree with $n$ edges and $j+1$ internal nodes has $n+1$ total nodes, thus $n-j$ leaves. From this it follows that $\left|\mathcal{T}_{n, j+1}^{(k)}\right|=\left|\mathcal{D}_{2 n, n-j}^{(k)}\right|$.

Next we provide a direct bijection from permutations in $\mathcal{A}_{n, j}^{(k)}$ to Dyck paths in $\mathcal{D}_{2 n, n-j}^{(k+1)}$. However, in subsequent sections of this paper we choose to use ordered trees to obtain enumeration results for the numbers $a_{n, j}^{(k)}$.

Theorem 5. For all $n \geq 1$ and all $j, k \geq 0$, there is a bijection $\phi_{n}: \mathcal{A}_{n, j}^{(k)} \rightarrow$ $\mathcal{D}_{2 n, n-j}^{(k+1)}$. In other words, $a_{n, j}^{(k)}$ is equal to the number of Dyck paths of length $2 n$ with $n-j$ peaks, and height less than or equal to $k+1$.

Proof. First we need to define the lifting of a path $P \in \mathcal{D}_{2 n}$ to path $L(P) \in \mathcal{D}_{2 n+2}$. Let $P=\left(p_{1}, \ldots, p_{2 n}\right)$ where each $p_{i}$ is either an up-step or a down-step. Then $L(P)$ is obtained from $P$ by appending an up-step at the start of $P$, and a down-step at the end of $P$. That is, $L(P)=$ $\left((1,1), p_{1}, \ldots, p_{2 n},(1,-1)\right)$. An example is shown in Figure 4. Also, if $P_{1} \in$ 

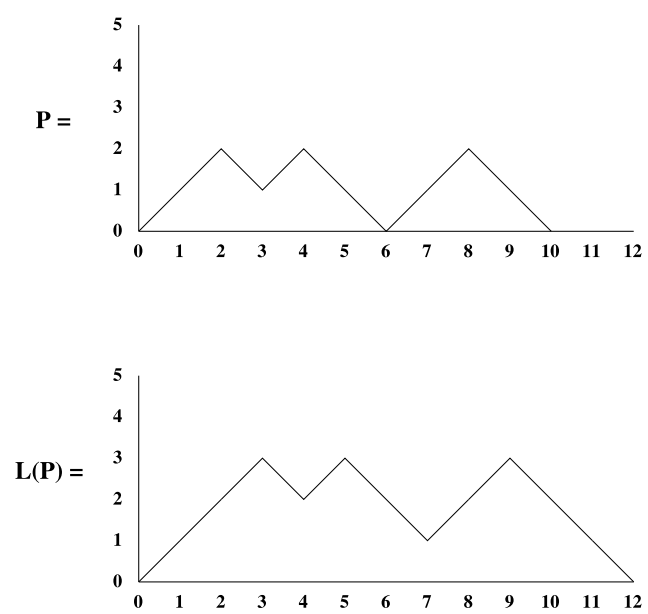

Figure 4: The lifting of a Dyck path.
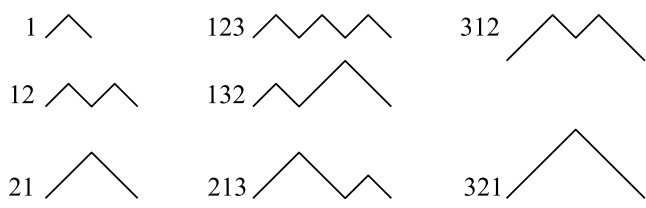

Figure 5: Values of $\phi_{n}$ up to $n=3$.

$\mathcal{D}_{2 n}$ and $P_{2} \in \mathcal{D}_{2 k}$, then we let $P_{1} P_{2} \in \mathcal{D}_{2 n+2 k}$ denote the path which starts with $P_{1}$ followed by $P_{2}$.

For $n=1$, we simply define $\phi_{1}(\sigma)=((1,1),(1,-1))$, i.e. and up-step followed by a down-step. For $n>1$ we define $\phi_{n}$ recursively by cases as follows.

Case 1. $\sigma_{n}=n$.

Then $\phi_{n}(\sigma)=P_{1} P_{2}$ where $P_{1}=\phi_{n-1}\left(\sigma_{1} \ldots \sigma_{n-1}\right)$ and $P_{2}=((1,1),(1,-1))$.

Case 2. $\sigma_{1}=n$.

Then $\phi_{n}(\sigma)=L\left(\phi_{n-1}\left(\sigma_{2} \ldots \sigma_{n}\right)\right)$.

Case 3. $\sigma_{i}=n$ where $1<i<n$. In this case, $\phi_{n}(\sigma)=P_{1} P_{2}$ where $P_{1}=\phi_{i-1}\left(\operatorname{red}\left(\sigma_{1} \ldots \sigma_{i-1}\right)\right)$ and $P_{2}=L\left(\phi_{n-i}\left(\operatorname{red}\left(\sigma_{i+1} \ldots \sigma_{n}\right)\right)\right)$.

The first few values of this map are pictured in Figure 5, where $\sigma$ is on the left and $\phi_{n}(\sigma)$ is on the right. 
Since $a_{n, j}^{(k)}=\left|\mathcal{T}_{n, j+1}^{(k+1)}\right|=\left|\mathcal{D}_{2 n, n-j}^{(k+1)}\right|$, it suffices to show that $\phi_{n}$ is welldefined and injective. We induct on $n$. The base case $n=1$ is obvious. Now let $n>1$ and assume the theorem holds for all $m<n$. Let $\sigma=\sigma_{1} \ldots \sigma_{n} \in$ $\mathcal{A}_{n, j}^{(k)}$, and let $\sigma_{i}=n$. Since $\sigma$ avoids the pattern 231, we have $\sigma_{1} \ldots \sigma_{i-1} \in$ $\mathcal{A}_{i-1}^{(k)}$, and $\operatorname{red}\left(\sigma_{i+1} \ldots \sigma_{n}\right) \in \mathcal{A}_{n-i}^{(k-1)}$ (see also the proof of Theorem 1). To show $\phi_{n}$ is well-defined, we consider the three cases for $i$ in the definition of $\phi_{n}$.

Case 1. $\sigma_{n}=n$.

In this case, $\phi_{n}(\sigma)=P_{1} P_{2}$ where $P_{1}=\phi_{n-1}\left(\sigma_{1} \ldots \sigma_{n-1}\right)$ and $P_{2}=((1,1),(1,-1))$. Since $n-1 \notin \operatorname{DES}(\sigma)$, we have $\sigma_{1} \ldots \sigma_{n-1} \in \mathcal{A}_{n-1, j}^{(k)}$. By the inductive hypothesis we have $P_{1} \in \mathcal{D}_{2 n-2, n-j-1}^{(k+1)}$. Since appending $P_{2}$ to $P_{1}$ increases the length by two, increases the number of peaks by one, and does not change the height, it follows that $P_{1} P_{2} \in \mathcal{D}_{2 n, n-j}^{(k+1)}$.

Case 2. $\sigma_{1}=n$.

In this case, $\phi_{n}(\sigma)=L\left(\phi_{n-1}\left(\sigma_{2} \ldots \sigma_{n}\right)\right)$. Since $1 \in \operatorname{DES}(\sigma)$, we have $\sigma_{2} \ldots \sigma_{n} \in \mathcal{A}_{n-1, j-1}^{(k-1)}$. By induction, $\phi_{n-1}\left(\sigma_{2} \ldots \sigma_{n}\right) \in \mathcal{D}_{2 n-2, n-j}^{(k)}$. Since lifting a path increases the height by one, increases the length by two, and adds no peaks, it follows that $L\left(\phi_{n-1}\left(\sigma_{2} \ldots \sigma_{n}\right)\right) \in \mathcal{D}_{2 n, n-j}^{(k+1)}$.

Case 3. $\sigma_{i}=n$ where $1<i<n$.

In this case, $\phi_{n}(\sigma)=P_{1} P_{2}$ where

$$
P_{1}=\phi_{i-1}\left(\operatorname{red}\left(\sigma_{1} \ldots \sigma_{i-1}\right)\right),
$$

and

$$
P_{2}=L\left(\phi_{n-i}\left(\operatorname{red}\left(\sigma_{i+1} \ldots \sigma_{n}\right)\right)\right) .
$$

Note that $\operatorname{red}\left(\sigma_{1} \ldots \sigma_{i-1}\right)=\sigma_{1} \ldots \sigma_{i-1}$. Since $i \in \operatorname{DES}(\sigma)$ we have $\sigma_{1} \ldots \sigma_{i-1} \in \mathcal{A}_{i-1, j_{1}}^{(k)}$, and $\operatorname{red}\left(\sigma_{i+1} \ldots \sigma_{n}\right) \in \mathcal{A}_{n-i, j_{2}}^{(k-1)}$, where $j_{1}+j_{2}+1=j$. Then

$$
\phi_{n-i}\left(\operatorname{red}\left(\sigma_{i+1} \ldots \sigma_{n}\right)\right) \in \mathcal{D}_{2 n-2 i, n-i-j_{2}}^{(k)},
$$

and

$$
P_{2}=L\left(\phi_{n-i}\left(\operatorname{red}\left(\sigma_{i+1} \ldots \sigma_{n}\right)\right)\right) \in \mathcal{D}_{2 n-2 i+2, n-i-j_{2}}^{(k+1)} .
$$

Also, $P_{1} \in \mathcal{D}_{2 i-2, i-1-j_{1}}^{(k+1)}$. It follows that $P_{1} P_{2} \in \mathcal{D}_{2 n, n-j_{1}-j_{2}-1}^{(k+1)}$ as desired since $n-j_{1}-j_{2}-1=n-j$.

This proves $\phi_{n}$ is well-defined. 
To prove injectivity let $\sigma, \pi \in \mathcal{A}_{n, j}^{(k)}$, and suppose $\sigma \neq \pi$. If $\sigma_{i}=\pi_{i}=n$ for some $i$, then for each case that $i$ falls into in the definition of $\phi_{n}$, one can easily use the inductive hypothesis to prove that $\phi_{n}(\sigma) \neq \phi_{n}(\pi)$. So assume $\sigma_{i_{1}}=n$ and $\pi_{i_{2}}=n$ where $i_{1}<i_{2}$. If $1 \leq i_{1}<i_{2} \leq n$, then it is easy to see that $\phi_{n}(\pi)$ hits the $x$-axis after $2 i_{2}-2$ steps while $\phi_{n}(\sigma)$ does not. Thus $\phi_{n}(\sigma) \neq \phi_{n}(\pi)$.

\section{Recursions and closed form expressions for $a_{n, j}^{(k)}$}

In this section we prove some recursions and closed form expressions for $a_{n, j}^{(k)}$. For certain cases of the values of $n, j, k$, we can find nice closed form expressions for these numbers (see also [4]). For the general case, it turns out that there is a closed form expression due to Kemp (see [5]) for a class of trees very closely related to $\mathcal{T}_{n, j}^{(k)}$. This formula can easily be translated to a closed form expression for $a_{n, j}^{(k)}$. We also prove the recurrence for $a_{n, j}^{(k)}$ from Theorem 2. We conclude this section by showing that this recurrence leads to the closed form expression for $a_{n, j}^{(k)}$ appearing in Corollary 1, which looks completely different from the formula due to Kemp.

Theorem 6 ([5, Theorem 1]). Let $h_{k}(n, j)$ be the number of ordered trees with $n$ nodes, $j$ leaves, and height $t^{1}$ less than or equal to $k-1$. Then $h_{k}(1, j)=$ $\delta_{j, 1}, h_{1}(n, j)=\delta_{n, j} \delta_{n, 1}$ where $\delta_{n, j}$ is Kronecker's symbol. For $k \geq 2$ and $n \geq 2$

$$
h_{k}(n, j)=N(n-1, j)-\left[Q_{1}(n, j, k)-2 Q_{0}(n, j, k)+Q_{-1}(n, j, k)\right],
$$

where

$$
Q_{a}(n, j, k)=\sum_{s \geq 1}\left(\begin{array}{c}
n-s(k-1)-2 \\
j+s+a-1
\end{array}\right)\left(\begin{array}{c}
n+s(k-1)-2 \\
j-s-a-1
\end{array}\right),
$$

and $N(n, j)$ are the Narayana numbers given by

$$
N(n, j)=\frac{1}{n}\left(\begin{array}{c}
n \\
j
\end{array}\right)\left(\begin{array}{c}
n \\
j-1
\end{array}\right) .
$$

Corollary 2. For all $n \geq 1$, and $j, k \geq 0$ we have

$$
a_{n, j}^{(k)}=h_{k+2}(n+1, n-j) .
$$

\footnotetext{
${ }^{1}$ In [5], the author uses the convention that the root is a vertex at level one, so we have translated this result to coincide with our definition of height.
} 
Proof. A tree with $n$ edges has $n+1$ nodes. An ordered tree with $n$ edges and $j+1$ internal nodes has $n+1-(j+1)=n-j$ leaves. Thus from Theorem 4 we have

$$
a_{n, j}^{(k)}=\left|\mathcal{T}_{n, j+1}^{(k+1)}\right|=h_{k+2}(n+1, n-j) .
$$

The Narayana numbers $N(n, j)$ appear in several combinatorial problems (see A001263 in the OEIS [10]). One interpretation is that $N(n, j)$ is equal to the number of Dyck paths of length $2 n$ with $j$ peaks. Another interpretation is that $N(n, j)$ is equal to the number of ordered trees with $n$ edges and $j$ leaves. Next we show that $a_{n, j}^{(k)}$ reduces to a Narayana number whenever $k \geq j$.

Corollary 3. For all $n>j \geq 1$, and for all $k \geq j$ we have

$$
a_{n, j}^{(k)}=N(n, n-j)=\frac{1}{n}\left(\begin{array}{c}
n \\
j
\end{array}\right)\left(\begin{array}{c}
n \\
j+1
\end{array}\right) .
$$

Proof. An ordered tree with $n$ edges and $j+1$ internal nodes has height less than or equal to $j+1$, and $n-j$ leaves. Thus whenever $k \geq j$ we have

$$
a_{n, j}^{(k)}=\left|\mathcal{T}_{n, j+1}^{(k+1)}\right|=N(n, n-j)=\frac{1}{n}\left(\begin{array}{c}
n \\
n-j
\end{array}\right)\left(\begin{array}{c}
n \\
n-j-1
\end{array}\right)=\frac{1}{n}\left(\begin{array}{c}
n \\
j
\end{array}\right)\left(\begin{array}{c}
n \\
j+1
\end{array}\right) .
$$

Note that in general $N(n, j)=N(n, n-j+1)$, i.e. the Narayana numbers are symmetric, and this follows from the symmetry of the binomial coefficients.

Our next goal is to establish the recurrence for $a_{n, j}^{(k)}$ appearing in Theorem 2. We accomplish this using a bijection to find a recurrence for trees with $n$ edges, $j$ leaves, and height less than or equal to $k$. Let $\mathcal{N}(n, j, k)$ denote the set of ordered trees with $n$ edges, $j$ leaves, and height less than or equal to $k$, and let $N(n, j, k)=|\mathcal{N}(n, j, k)|$. In other words, $N(n, j, k)$ are the Narayana numbers refined by height. For example, $\mathcal{N}(k, k, 1)$ consists of a single tree $T$ of height 1 whose root has $k$ children. For convenience, we let $\mathcal{N}(0,0, k)$ be the set containing the tree with one vertex and no edges, hence $N(0,0, k)=1$ for all $k \geq 0$. Note that in terms of Dyck paths we have $N(n, j, k)=\left|\mathcal{D}_{2 n, j}^{(k)}\right|$, i.e. the number of Dyck paths of length $2 n$ with $j$ peaks and height less than or equal to $k$. We find a recurrence for $N(n, j, k)$, which can easily be translated into a recurrence for $a_{n, j}^{(k)}$. Before we prove this theorem, we need the following definition and proposition. 
Definition 1. Let $W_{n}(i, j)$ be the set of weak compositions $\left(p_{1}, p_{2}, \ldots, p_{i} ; m_{1}, m_{2}, \ldots, m_{j}\right)$ in $\mathbb{N}^{i+j}$ such that

(i) $p_{r} \geq 1$ for $r=1,2, \ldots i$,

(ii) $m_{r} \geq 0$ for $r=1,2, \ldots j$, and

(iii) $\left(\sum_{r=1}^{i} p_{r}\right)+\left(\sum_{r=1}^{j} m_{r}\right)=n$.

In other words, $W_{n}(i, j)$ is the set of weak compositions of $n$ with $i+j$ parts where the first $i$ parts are positive.

Proposition 1. For all $n, i, j \geq 0$ we have $\left|W_{n}(i, j)\right|=\left(\begin{array}{c}n+j-1 \\ i+j-1\end{array}\right)$.

Proof. Let $p_{r}^{\prime}=p_{r}-1$ for $r=1,2, \ldots, i$. Then

$$
\left(p_{1}, p_{2}, \ldots, p_{i} ; m_{1}, m_{2}, \ldots, m_{j}\right) \in W_{n}(i, j)
$$

if and only if

$$
\left(p_{1}^{\prime}, p_{2}^{\prime} \ldots, p_{i}^{\prime} ; m_{1}, m_{2}, \ldots, m_{j}\right) \in W_{n-i}(0, i+j)
$$

$W_{n}(0, k)$ is simply the number of weak compositions of $n$ into $k$ parts, and $\left|W_{n}(0, k)\right|=\left(\begin{array}{c}n+k-1 \\ k-1\end{array}\right)($ see $[11])$. Thus

$$
\left|W_{n}(i, j)\right|=\left|W_{n-i}(0, i+j)\right|=\left(\begin{array}{c}
n+j-1 \\
i+j-1
\end{array}\right) .
$$

For convenience we recall Theorem 2 before providing its proof: for all $k \geq 2$ and for all $n \geq j \geq 1$ we have

$$
a_{n, n-j}^{(k-1)}=N(n, j, k)=\sum_{i=0}^{n-j} N(n-j, i, k-1)\left(\begin{array}{c}
2 n-j-i \\
2 n-2 j
\end{array}\right),
$$

where $N(n, j, 1)=1$ if $n=j$, and is zero otherwise.

\section{Proof of Theorem 2.}

The fact that $a_{n, n-j}^{(k-1)}=N(n, j, k)$ follows from Theorem 4 , so it remains to prove the second equality in Theorem 2. For this we construct a map

$$
s: \bigcup_{i=0}^{n-j} \mathcal{N}(n-j, i, k-1) \times W_{j}(i, 2 n-2 j-i+1) \rightarrow \mathcal{N}(n, j, k),
$$

which we will show is a bijection. 


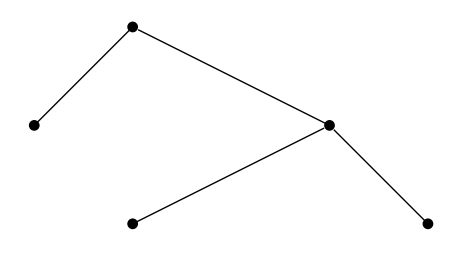

$T, \quad c=(1,2,2 ; 1,2,0,0,1,1)$

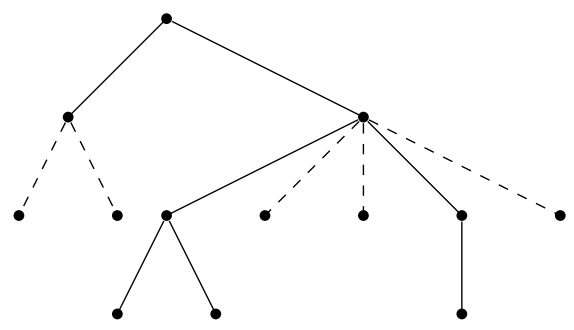

$U_{2}, \quad c=(1,2, \mathbf{2} ; \mathbf{1}, \mathbf{2}, \mathbf{0}, 0,1,1)$

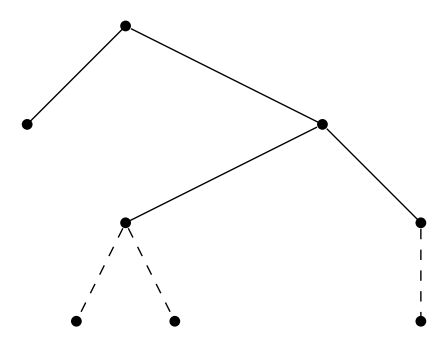

$U_{3}, \quad c=(\mathbf{1}, \mathbf{2}, 2 ; 1,2,0,0,1,1)$

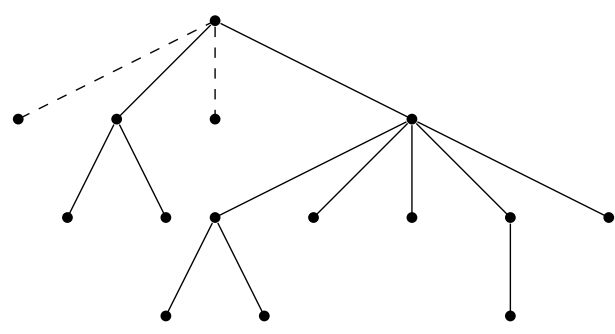

$U_{1}, \quad c=(1,2,2 ; 1,2,0, \mathbf{0}, \mathbf{1}, \mathbf{1})$

Figure 6: An example of the map $s$.

Informally, given

$$
(T, c) \in \mathcal{N}(n-j, i, k-1) \times W_{j}(i, 2 n-2 j-i+1),
$$

one obtains $s(T, c)$ as follows: visit the vertices of $T$ from right to left and bottom to top, at the leaves add edges according to the positive parts of $c$, and at the internal nodes add edges between pairs of children according the nonnegative parts of $c$. For example let $n=14, j=10, k=3, i=3$, and let $T \in \mathcal{N}(4,3,2)$ be the tree in the upper left of Figure 6. Each tree $U_{h}$ for $h=1,2,3$ in Figure 6 shows the added edges (dashed) obtained by visiting the vertices at level $h$, and the parts of $c$ determining the number of edges added are in bold. The output of this map is $s(T, c)=U_{1}$.

A formal description of $s$ is as follows. Let $T \in \mathcal{N}(n-j, i, k-1)$, and let

$$
c=\left(l_{1}, \ldots, l_{i} ; n_{1}, \ldots, n_{2 n-2 j-i+1}\right) \in W_{j}(i, 2 n-2 j-i+1)
$$

for some $i$ such that $0 \leq i \leq n-j$. We describe $s(T, c)$ via a composition of 
maps, $s=s_{1} \circ s_{2} \circ \cdots \circ s_{k}$, where each $s_{h}$ implicitly depends on $c$. Let

$$
U_{h}=s_{h} \circ s_{h+1} \circ \cdots \circ s_{k}(T)
$$

for $1 \leq h \leq k$, and set $U_{k+1}=T$, thus $s(T, c)=U_{1}$. Construct an ordered tree $U_{h-1}=s_{h-1}\left(U_{h}\right)$ by visiting the vertices at level $h-1$ of $U_{h}$ from right to left, and (possibly) adding edges to each vertex $x$ as follows:

- If $x$ is the $p^{\text {th }}$ leaf visited in the process of applying $s_{h-1}, s_{h}, \ldots, s_{k}$ to $T$, then attach the unique tree from $\mathcal{N}\left(l_{p}, l_{p}, 1\right)$ as a subtree below $x$.

- Suppose $x$ is an internal node. Let nc $(x)$ denote the number of children of $x$ (i.e. $\operatorname{nc}(x)+1=\operatorname{deg} x$ ), and suppose $\mathrm{nc}(x)=d$. Attach the tree from $\mathcal{N}\left(n_{r}, n_{r}, 1\right)$ to the right of the rightmost edge below $x$ where $r=1+\sum(\operatorname{nc}(y)+1)$ and the sum is over all internal nodes previously visited in the process of applying $s_{h-1}, s_{h}, \ldots, s_{k}$ to $T$. Then for $m=$ $1,2, \ldots, d$, attach the tree from $\mathcal{N}\left(n_{r+m}, n_{r+m}, 1\right)$ to the left of the $m$-th rightmost edge below $x$.

First we show that $s$ is well-defined. Note that $T$ has $n-j$ edges and $c$ is a weak composition of $j$. Thus applying $s$ will add $j$ edges to $T$, so $s(T, c)$ has $n$ edges. Since $l_{1}, \ldots, l_{i}$ are all positive, every leaf of $T$ has edges added to it, and is therefore not a leaf in $s(T, c)$. On the other hand, every edge added to $T$ creates a leaf, so $s(T, c)$ has $j$ leaves. Since $T$ has height less than or equal to $k-1$, it is clear that $s(T, c)$ has height less than or equal to $k$. Furthermore, $T$ has $i$ leaves and the first $i$ parts of $c$ are positive. We also need to check that $c$ has the appropriate number of parts for adding edges to internal nodes. This follows from the fact that

$$
\begin{aligned}
\sum_{\substack{x \text { is an internal } \\
\text { node of } T}}(1+\operatorname{nc}(x)) & =\mid\{\text { internal nodes of } T\} \mid+\sum_{x \text { is an internal }} \operatorname{nc}(x) \\
& =\mid\{\text { vede of } T \\
& =(n-j+1)-i+(n-j) \\
& =2 n-2 j-i+1 .
\end{aligned}
$$

Next we describe the inverse map of $s$, which we denote by $f$. We have chosen the letter $s$ to correspond to spring, since the tree "grows" edges during this map. And the letter $f$ corresponds to fall since we will remove edges during this map. Let $T \in \mathcal{N}(n, j, k)$.

Given $T \in \mathcal{N}(n, j, k)$ let $f(T)=(V, c)$. Informally, one obtains the tree $V$ as follows: visit the vertices of $T$ from left to right and top to bottom, 


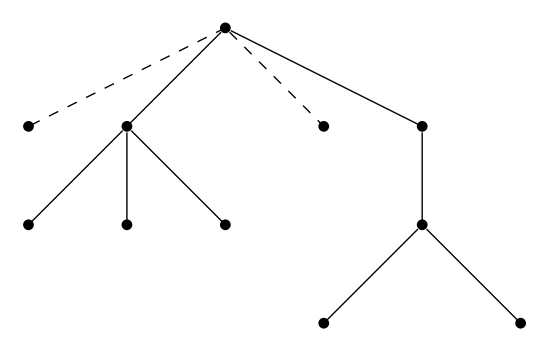

$V_{0}, \quad c_{0}=\emptyset$

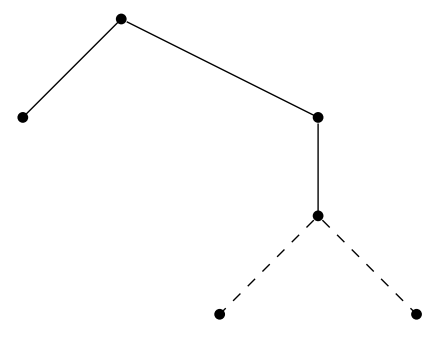

$V_{2}, \quad c_{2}=(3 ; 0,0,0,1,1)$

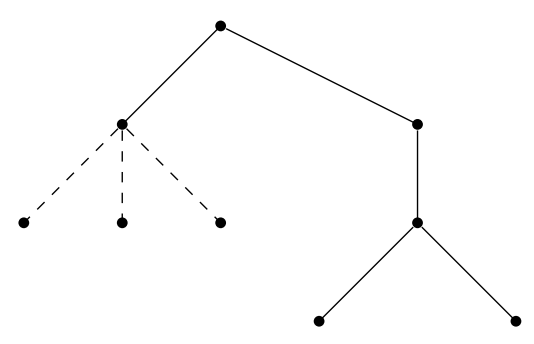

$V_{1}, \quad c_{1}=(0,1,1)$

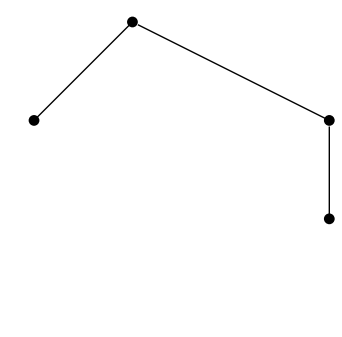

$V_{3}, \quad c_{3}=(2,3 ; 0,0,0,1,1)$

Figure 7: An example of the map $f$.

removing all single edges below each vertex. If the current vertex has no grandchildren, then record the number of edges removed as a positive part of $c$. If the current vertex does have grandchildren, then record the number of edges removed between each pair of non-removable children (i.e. children of the current vertex who themselves have children) as a nonnegative part of $c$.

For example let $V_{0} \in \mathcal{N}(10,7,3)$ be the tree in the upper left of Figure 7. Each tree $V_{h}$ for $h=0,1,2$ in Figure 7 shows the edges (dashed) to be removed at height $h+1$. The output is of this map is $f\left(V_{0}\right)=\left(V_{3}, c_{3}\right)$.

Formally, we describe $f(T)$ via a composition of maps $f=f_{k} \circ f_{k-1} \circ$ $\cdots \circ f_{1}$. Let

$$
\left(V_{h}, c_{h}\right)=f_{h} \circ f_{h-1} \circ \cdots \circ f_{1}(T)
$$

for $1 \leq h \leq k$, where $V_{h}$ is an ordered tree and $c_{h}$ is a weak composition, and let $\left(V_{0}, c_{0}\right)=(T, \emptyset)$. Note that $f(T)=\left(V_{k}, c_{k}\right)$. Construct $\left(V_{h+1}, c_{h+1}\right)=$ $f_{h+1}\left(V_{h}, c_{h}\right)$ by visiting the vertices at level $h$ of $V_{h}$ from left to right, and removing all single edges below each vertex. The weak composition $c_{h+1}$ 
is obtained from $c_{h}$ by recording at each vertex $x$, the numbers of edges removed as follows:

- If $x$ has $p$ children and all subtrees below $x$ have height one (in other words $x$ has no grandchildren), then append a $p$ to the beginning of the positive parts of $c_{h}$.

- Suppose $x$ has subtrees below it of height greater than one. Call $Y$ an immediate subtree of $x$ if the root of $Y$ is $x, Y$ has exactly one vertex $y$ of level one, and $Y$ contains all edges and vertices below $y$. Suppose $x$ has immediate subtrees $Y_{1}, Y_{2}, \ldots, Y_{d}$ (from left to right) of height greater than or equal to 2 . Let $r_{1}$ be the number of single edges below $x$ and to the left of $Y_{1}$. For $m=2,3, \ldots, d$, let $r_{m}$ equal the number of single edges below $x$ between $Y_{m-1}$ and $Y_{m}$. Let $r_{d+1}$ be the number of single edges below $x$ and to the right of $Y_{d}$. Then append the parts $\left(r_{d+1}, r_{d}, \ldots, r_{1}\right)$ to the beginning of the nonnegative parts of $c_{h}$.

Next we show that $f$ is well-defined. When applying $f_{h}$, we never visit a vertex which is a leaf since such a vertex would have been removed when applying $f_{h-1}$. Since we remove from $T$ precisely all edges which have a leaf at the bottom, we see that $V_{k}$ has $n-j$ edges. Since $V_{k}$ has $n-j$ edges, the number of leaves of $V_{k}$ is less than or equal to $n-j$. Clearly, the height of $V_{k}$ is one less than the height of $T$. Thus $V_{k} \in \mathcal{N}(n-j, i, k-1)$ for some $0 \leq i \leq n-j$.

Since the map $f$ removes $j$ edges from $T$ and $c_{k}$ records the total number of edges removed, $c_{k}$ is a weak composition of $j$. A leaf of $V_{k}$ is created only when we visit a vertex with only single edges below. The number of such edges is recorded as a positive part in the weak composition $c_{k}$. So if $V_{k}$ has $i$ leaves, then $c_{k}$ has $i$ positive parts. Lastly, the total number of parts (positive and nonnegative) of $c_{k}$ is given by

$$
\begin{aligned}
\sum_{\substack{x \text { is a } \\
\text { vertex of } V_{k}}}(1+\mathrm{nc}(x)) & =\mid\left\{\text { vertices of } V_{k}\right\}|+|\left\{\text { edges of } V_{k}\right\} \mid \\
& =(n-j+1)+(n-j) \\
& =2 n-2 j+1
\end{aligned}
$$

thus $c_{k}$ has $2 n-2 j+1-i$ nonnegative parts.

It is clear by construction that $f$ is the inverse of $s$.

The Theorem now follows from the fact that (see Proposition 1)

$$
\left|W_{j}(i, 2 n-2 j-i+1)\right|=\left(\begin{array}{c}
2 n-j-i \\
2 n-2 j
\end{array}\right) .
$$


This completes the proof of Theorem 2 .

For convenience we state Theorem 2 in terms of $a_{n, j}^{(k)}$.

Corollary 4. For all $k \geq 1$ and $n>j$, we have

$$
a_{n, j}^{(k)}=\sum_{i=0}^{j} a_{j, i}^{(k-1)}\left(\begin{array}{c}
n+i \\
2 j
\end{array}\right),
$$

where $a_{0,0}^{(k-1)}:=1$ for all $k \geq 1$.

The recurrence in Corollary 4 can be iterated to obtain a closed form expression for $a_{n, j}^{(k)}$. Indeed, $a_{j, i}^{(0)}$ is the number of permutations in $S_{j}(231)$ with $i$ descents and no drops. Since the identity permutation is the only permutation with no drops, we see that $a_{j, i}^{(0)}=1$ if $i=0$, and $a_{j, i}^{(0)}$ is zero otherwise. Hence

$$
a_{n, j}^{(1)}=\sum_{i=0}^{j} a_{j, i}^{(0)}\left(\begin{array}{c}
n+i \\
2 j
\end{array}\right)=\left(\begin{array}{c}
n \\
2 j
\end{array}\right),
$$

as expected (see Theorem 3). We iterate to obtain the following formulas, and in each case the formula holds for all $n>j \geq 0$.

$$
\begin{aligned}
& a_{n, j}^{(2)}=\sum_{j \geq i \geq 0} a_{j, i}^{(1)}\left(\begin{array}{c}
n+i \\
2 j
\end{array}\right)=\sum_{j \geq i \geq 0}\left(\begin{array}{c}
j \\
2 i
\end{array}\right)\left(\begin{array}{c}
n+i \\
2 j
\end{array}\right) . \\
& a_{n, j}^{(3)}=\sum_{j \geq i_{2} \geq i_{1} \geq 0}\left(\begin{array}{c}
i_{2} \\
2 i_{1}
\end{array}\right)\left(\begin{array}{c}
j+i_{1} \\
2 i_{2}
\end{array}\right)\left(\begin{array}{c}
n+i_{2} \\
2 j
\end{array}\right) . \\
& a_{n, j}^{(4)}=\sum_{j \geq i_{3} \geq i_{2} \geq i_{1} \geq 0}\left(\begin{array}{c}
i_{2} \\
2 i_{1}
\end{array}\right)\left(\begin{array}{c}
i_{3}+i_{1} \\
2 i_{2}
\end{array}\right)\left(\begin{array}{c}
j+i_{2} \\
2 i_{3}
\end{array}\right)\left(\begin{array}{c}
n+i_{3} \\
2 j
\end{array}\right) . \\
& a_{n, j}^{(5)}=\sum_{j \geq i_{4} \geq i_{3} \geq i_{2} \geq i_{1} \geq 0}\left(\begin{array}{c}
i_{2} \\
2 i_{1}
\end{array}\right)\left(\begin{array}{c}
i_{3}+i_{1} \\
2 i_{2}
\end{array}\right)\left(\begin{array}{c}
i_{4}+i_{2} \\
2 i_{3}
\end{array}\right)\left(\begin{array}{c}
j+i_{3} \\
2 i_{4}
\end{array}\right)\left(\begin{array}{c}
n+i_{4} \\
2 j
\end{array}\right) .
\end{aligned}
$$

A pattern emerges, giving us a formula for $a_{n, j}^{(k)}$ and $N(n, j, k)$. For convenience we recall Corollary 1 , and then provide its proof.

For $n \geq j \geq 0$ and $k \geq 3$ we have

$$
N(n, j, k)=a_{n, n-j}^{(k-1)}=\sum_{n-j \geq i_{k-2} \geq \cdots \geq i_{1} \geq 0}\left(\prod_{m=0}^{k-2}\left(\begin{array}{c}
i_{m+2}+i_{m} \\
2 i_{m+1}
\end{array}\right)\right),
$$

where $i_{0}:=0, \quad i_{k-1}:=n-j, \quad i_{k}:=n$. 


\section{Proof of Corollary 1.}

Proof. We prove that for all $n, j \geq 0$ and all $k \geq 2$ we have

$$
a_{n, j}^{(k)}=\sum_{j \geq i_{k-1} \geq \cdots \geq i_{1} \geq 0}\left(\prod_{m=0}^{k-1}\left(\begin{array}{c}
i_{m+2}+i_{m} \\
2 i_{m+1}
\end{array}\right)\right),
$$

where $i_{0}:=0, \quad i_{k}:=j, \quad i_{k+1}:=n$. The corollary is then implied by Theorem 4 .

Induct on $k$. The base case $k=2$ follows from Corollary 4 . Now let $k \geq 3$ and assume the result holds for $k-1$. Then from Corollary 4 we have

$$
a_{n, j}^{(k)}=\sum_{j \geq p_{k-1} \geq 0} a_{j, p_{k-1}}^{(k-1)}\left(\begin{array}{c}
n+p_{k-1} \\
2 j
\end{array}\right) .
$$

Use the induction hypothesis to substitute an expression for $a_{j, p_{k-1}}^{(k-1)}$.

$$
\begin{aligned}
a_{n, j}^{(k)}=\sum_{\substack{j \geq p_{k-1} \geq 0 \\
p_{k-1} \geq i_{k-2} \geq \cdots \geq i_{1} \geq 0}}\left(\prod_{m=0}^{k-4}\left(\begin{array}{c}
i_{m+2}+i_{m} \\
2 i_{m+1}
\end{array}\right)\right)\left(\begin{array}{c}
p_{k-1}+i_{k-3} \\
2 i_{k-2}
\end{array}\right)\left(\begin{array}{c}
j+i_{k-2} \\
2 p_{k-1}
\end{array}\right) \\
\times\left(\begin{array}{c}
n+p_{k-1} \\
2 j
\end{array}\right) .
\end{aligned}
$$

The result now follows from replacing $p_{k-1}$ with $i_{k-1}$.

Note that for $k \geq 1, e_{n, j}^{(k)}=a_{n, j}^{(k)}-a_{n, j}^{(k-1)}$ is the number of permutations $\sigma \in S_{n}(231)$ such maxdrop $(\sigma)=k$. Observing that the $i_{1}=0$ term of $a_{n, j}^{(k)}$ is just the expression for $a_{n, j}^{(k-1)}$, we immeditately have the following corollary of Corollary 1.

Corollary 5. For all $k \geq 2$ and $n>j \geq 0$,

$$
e_{n, j}^{(k)}=\sum_{j \geq i_{k-1} \geq \cdots \geq i_{1} \geq 1}\left(\prod_{m=0}^{k-1}\left(\begin{array}{c}
i_{m+2}+i_{m} \\
2 i_{m+1}
\end{array}\right)\right) \text {, }
$$

where $i_{0}:=0, i_{k}:=j, \quad i_{k+1}:=n$.

For example, when $k=2$ and $j=2$ or $j=3$, there is only one term in the sum so that $e_{n, 2}^{(2)}=\left(\begin{array}{c}n+1 \\ 4\end{array}\right)$ and $e_{n, 3}^{(2)}=3\left(\begin{array}{c}n+1 \\ 6\end{array}\right)$. Similarly, when $k=2$ and $j=4$ or $j=5$, there are only two terms in the sum so that $e_{n, 4}^{(2)}=\left(\begin{array}{c}n+2 \\ 8\end{array}\right)+6\left(\begin{array}{c}n+1 \\ 8\end{array}\right)$ 
and $e_{n, 5}^{(2)}=5\left(\begin{array}{c}n+2 \\ 10\end{array}\right)+10\left(\begin{array}{c}n+1 \\ 10\end{array}\right)$. Also one can show that in the sum for $e_{n, j}^{(j)}$, there is only one term that gives a nonzero contribution, namely, $i_{s}=s$ for $s=1, \ldots, j$ so that $e_{n, j}^{(j)}=\left(\begin{array}{c}n+j-1 \\ 2 j\end{array}\right)$. It would be interesting to give a simple direct combinatorial explanation for any of these facts.

\section{Resulting identities}

In the previous section we proved that the set of permutations in $S_{n}(231)$ with $j$ descents and maximum drop less than or equal to $k$ is in bijective correspondence with the set of ordered trees with $n$ edges, $j+1$ internal nodes, and height less than or equal to $k+1$. We also found two seemingly different closed form expressions for the number of such trees: one due to Kemp [5] (Theorem 6 and Corollary 2), and another resulting from iterating our recurrence (Corollary 1). This leads to some remarkable identities.

Theorem 7. For $n \geq 1$ and $j \geq 0$ we have

$$
a_{n, j}^{(1)}=h_{3}(n+1, n-j) .
$$

Consequently

$$
\left(\begin{array}{c}
n \\
2 j
\end{array}\right)=N(n, j+1)-\left[\widetilde{Q}_{1}(n, j, 3)-2 \widetilde{Q}_{0}(n, j, 3)+\widetilde{Q}_{-1}(n, j, 3)\right],
$$

where

$$
N(n, j+1)=\frac{1}{n}\left(\begin{array}{c}
n \\
j+1
\end{array}\right)\left(\begin{array}{c}
n \\
j
\end{array}\right)
$$

and

$$
\widetilde{Q}_{a}(n, j, 3)=\sum_{s \geq 1}\left(\begin{array}{c}
n-2 s-1 \\
j-3 s-a
\end{array}\right)\left(\begin{array}{c}
n+2 s-1 \\
j+3 s+a
\end{array}\right) .
$$

Proof. First note that (7) is just a special case of Corollary 2 with $k=1$.

The left hand side of (8) follows from Theorem 3. While the right hand side of (8) follows from Theorem 6 , noting that

$$
N(n, n-j)=N(n, j+1),
$$

and

$$
Q_{a}(n+1, n-j, 3)=\sum_{s \geq 1}\left(\begin{array}{c}
n-2 s-1 \\
n-j+s+a-1
\end{array}\right)\left(\begin{array}{c}
n+2 s-1 \\
n-j-s-a-1
\end{array}\right)
$$




$$
=\widetilde{Q}_{a}(n, j, 3)
$$

using the symmetry of the Narayana numbers and the binomial coefficients.

Remark 2 . If $j=0,1$, then $\widetilde{Q}_{a}(n, j, 3)=0$ for $a=-1,0,1$, and equation (8) follows immediately.

However, for $j \geq 2$ there will be nonzero contributions from $\widetilde{Q}_{a}(n, j, 3)$ for $a \leq j-3$. For example, if $j=2$ then

$$
\widetilde{Q}_{-1}(n, 2,3)=\sum_{s \geq 1}\left(\begin{array}{c}
n-2 s-1 \\
2-3 s+1
\end{array}\right)\left(\begin{array}{l}
n+2 s-1 \\
2+3 s-1
\end{array}\right)=\left(\begin{array}{c}
n+1 \\
4
\end{array}\right),
$$

and the right hand side of (8) becomes

$$
\begin{aligned}
& \frac{1}{n}\left(\begin{array}{l}
n \\
3
\end{array}\right)\left(\begin{array}{l}
n \\
2
\end{array}\right)-\left(\begin{array}{c}
n+1 \\
4
\end{array}\right)=\frac{1}{n}\left(\begin{array}{l}
n \\
3
\end{array}\right)\left(\begin{array}{l}
n \\
2
\end{array}\right)-\left(\begin{array}{l}
n \\
4
\end{array}\right)-\left(\begin{array}{l}
n \\
3
\end{array}\right) \\
= & \left(\begin{array}{l}
n \\
3
\end{array}\right)\left[\frac{n-1}{2}-1\right]-\left(\begin{array}{l}
n \\
4
\end{array}\right)=2\left(\begin{array}{l}
n \\
3
\end{array}\right)\left[\frac{n-3}{4}\right]-\left(\begin{array}{l}
n \\
4
\end{array}\right)=\left(\begin{array}{l}
n \\
4
\end{array}\right)
\end{aligned}
$$

as expected.

More generally, we can use Corollary 1 when $k \geq 2$.

Theorem 8. For $n \geq 1, j \geq 0$, and $k \geq 2$ we have

$$
\begin{aligned}
& \sum_{j \geq i_{k-1} \geq \cdots \geq i_{1} \geq 0}\left(\prod_{m=0}^{k-1}\left(\begin{array}{c}
i_{m+2}+i_{m} \\
2 i_{m+1}
\end{array}\right)\right) \\
= & N(n, j+1)-\left[\widetilde{Q}_{1}(n, j, k+2)-2 \widetilde{Q}_{0}(n, j, k+2)+\widetilde{Q}_{-1}(n, j, k+2)\right],
\end{aligned}
$$

where

$$
N(n, j+1)=\frac{1}{n}\left(\begin{array}{c}
n \\
j+1
\end{array}\right)\left(\begin{array}{c}
n \\
j
\end{array}\right)
$$

and

$$
\widetilde{Q}_{a}(n, j, k+2)=\sum_{s \geq 1}\left(\begin{array}{c}
n-(k+1) s-1 \\
j-(k+2) s-a
\end{array}\right)\left(\begin{array}{c}
n+(k+1) s-1 \\
j+(k+2) s+a
\end{array}\right) .
$$

Proof. From Corollary 2 we have

$$
a_{n, j}^{(k)}=h_{k+2}(n+1, n-j) .
$$


The left hand side of Theorem 8 follows from Corollary 1. And the right hand side of Theorem 8 follows from Theorem 6, noting that

$$
\begin{aligned}
Q_{a}(n+1, n-j, k+2) & =\sum_{s \geq 1}\left(\begin{array}{c}
n-(k+1) s-1 \\
n-j+s+a-1
\end{array}\right)\left(\begin{array}{c}
n+(k+1) s-1 \\
n-j-s-a-1
\end{array}\right) \\
& =\widetilde{Q}_{a}(n, j, k+2) .
\end{aligned}
$$

\section{References}

[1] F. Chung, A. Claesson, M. Dukes and R. Graham (2010). Descent polynomials for permutation with bounded drop size. European J. Combin. 31 no. 7, 1853-1867. MR2673024

[2] P. Flajolet and R. Sedgewick (2009). Analytic combinatorics. Cambridge University Press, Cambridge. MR2483235

[3] M. Hyatt (2013). Descent polynomials for $k$ bubble-sortable permutations of type B. European J. Combin. 34 no. 7, 1177-1191. MR3055231

[4] M. Hyatt and J. Remmel (2012). The classification of 231-avoiding permutations by descents and maximum drop. Preprint arXiv:1208.1052 [math.CO]

[5] R. Kemp (1983). The average height of planted plane trees with $m$ leaves. J. Combin. Theory Ser. B 34 no. 2, 191-208. MR0703604

[6] S. Kitaev (2011). Patterns in permutations and words, (with a foreword by Jeffrey B. Remmel). Springer, Heidelberg. MR3012380

[7] D. Knuth (1969). The Art of Computer Programming, Vol. 1 Fundamental Algorithms. Addison-Wesley, Reading, MA. MR0286317

[8] D. Knuth (1998). The Art of Computer Programming, Vol. 3 Sorting and Searching, 2nd ed. Addison-Wesley, Reading, MA. MR3077154

[9] A. Micheli and D. Rossin (2006). Edit distance between unlabaled ordered trees. Theor. Inform. Appl. 40 no. 4, 593-609. MR2277052

[10] N. J. A. Sloane. OEIS Foundation Inc. (2011). The On-Line Encyclopedia of Integer Sequences, http://oeis.org/A001263.

[11] R. P. Stanley (2012). Enumerative Combinatorics, Vol. 1, 2nd ed. Cambridge University Press, Cambridge. MR2868112

[12] R. P. Stanley (1999). Enumerative Combinatorics, Vol. 2. Cambridge University Press, Cambridge. MR1676282 
MATTHEW HyatT

Department of Mathematics

University of CALIFornia

SAN DIEgo

LA Jolla, CA 92093-0112

USA

E-mail address: matthewdhyatt@gmail.com

JEFFREY REMMEL

Department of Mathematics

University of CALIFORNIA

SAN DIEGO

LA Jolla, CA 92093-0112

USA

E-mail address: jremmel@ucsd.edu

RECEIVED 20 JANUARY 2014 